\title{
Giant Right Coronary Artery Aneurysm in the Setting of Acute Coronary Syndrome, A Case Report
}

\author{
salah altarabsheh ${ }^{1}$, Salil Deo ${ }^{2}$, Abeer Rababa'h ${ }^{3}$, Sakher Mayayah ${ }^{4}$, Mohammad \\ Alkhasawneh $^{4}$, Dalia Alhabahbeh ${ }^{5}$, and Yagthan Obeidat ${ }^{6}$ \\ ${ }^{1}$ Queen Alia Heart Institute \\ ${ }^{2}$ Louis Stokes Cleveland Medical Center \\ ${ }^{3}$ Jordan University of Science and Technology \\ ${ }^{4}$ Affiliation not available \\ ${ }^{5}$ King Hussein Medical Center \\ ${ }^{6}$ Almana General Hospitals
}

May 6, 2020

\begin{abstract}
Abstract Giant right coronary artery aneurysm is a rare coronary artery pathology. We describe a 45-year-old gentleman who presented with unstable angina of recent onset. Diagnostic work up including chest CT angiography and left heart catheterization demonstrated 3-vessel coronary artery disease with giant proximal right coronary artery aneurysm. In the view of the severity of the coronary artery disease and the risk of rupture associated with the giant right coronary artery aneurysm and the clinical presentation, patient was successfully treated by coronary artery bypass surgery. During this procedure, the right coronary artery aneurysm was ligated at both inflow and outflow. Patient recovered well and was discharged home.
\end{abstract}

\section{Introduction:}

Coronary artery aneurysm is a rare entity; with an incidence among patients undergoing coronary angiography that varies from $1.5-5 \%(1,2)$. Although the exact definition of giant coronary artery aneurysm is lacking, however it is usually referred to as a dilated segment exceeding 4 times the diameter of the largest coronary artery or an aneurysm exceeding $8 \mathrm{~mm}$ in diameter (3)

Treatment options are percutaneous coronary angioplasty or surgery. Here, we present our operative approach in treating a giant aneurysm of the right coronary artery with double ligation and distal bypass grafting.

\section{Case Report:}

A 47-year-old Hypertensive, non-insulin dependent diabetic gentleman presented to the emergency department with recurrent attacks of chest pain suggesting unstable angina. His diagnostic workup included blood tests, Echocardiography, left heart catheterization which demonstrated a huge dilatation of the right coronary artery with proximal and distal stenosis (Figure IA), and multi-vessel coronary artery disease involving the left coronary system (Figure IB) and Contrast-enhanced chest CT (Figure IC). In the present case, our patient had 3 vessel coronary artery disease with strong symptoms, and also having right sided dominant circulation with a posterior descending artery measuring $1.5 \mathrm{~mm}$ in diameter, and no major branch such as marginal or sinoatrial arteries could be demonstrated to take off from the giant right coronary aneurysm by examining various views of the right coronary angiogram. In the view of the patient presentation and the presence of multi-vessel coronary artery disease and the presence of the giant right coronary artery aneurysm, 
decision was taken to offer our patient the option of coronary artery bypass grafting with ligation of the right coronary artery aneurysm.

Surgery was performed via median sternotomy; the left internal thoracic artery (LITA) and left great saphenous vein (GSV) were harvested as conduits. Routine Cardiopulmonary bypass and cardioplegia was used. The right coronary artery aneurysm was isolated proximally and distally. It was then ligated with Proline $4 / 0$ threads (Figure I D), Extreme caution was taken not to excessively mobilize the giant aneurysm in order to prevent distal embolization of calcium or debris into the distal right coronary artery territory. Luckily, there were no branches in the RCA at the location of the aneurysm, and distal to the ligation, the posterior descending branch of the RCA was grafted with saphenous vein. Remaining part of the procedure was completed and patient weaned uneventfully off cardiopulmonary bypass with good ventricular function and in normal sinus rhythm. Dual antiplatelet therapy was initiated in the second day postoperatively.

\section{Discussion}

Giant coronary artery aneurysms are extremely rare; is an extremely rare condition, with a reported incidence of $0.02 \%$, among patients undergoing coronary angiography with a predilection to the right coronary artery, followed by the left anterior descending, left main coronary artery and circumflex artery (4). An increasing incidence had been reported after the wide spread utilization of drug eluting stents, possibly due to the arterial wall damage during balloon angioplasty or during stent deployment (5). Majority of coronary artery aneurysms are asymptomatic and discovered during diagnostic angiography, however giant coronary artery aneurysms may present with complications such as rupture, thrombo-embolic phenomena, arrhythmias, vasospasm or rarely it may form a communicating fistula with a heart chamber depending on the size, extent of involvement and status of the other coronary arteries (6).

The pathogenesis of such coronary aneurysm is due to weakness of the arterial wall of the coronary artery which leads to arterial dilatation. Multiple etiologies have been linked to this pathology including atherosclerosis, Kawasaki disease, vasculitis, hypercholesteremia and blunt trauma (7). In our case, atherosclerosis is the most probable etiology, since our patient is having strong risk factors for atherosclerosis including, systemic hypertension, heavy smoking, uncontrolled diabetes mellites and abnormally elevated lipid profile readings $(\mathrm{LDL}=139 \mathrm{mg} / \mathrm{dl}$, Total Cholesterol $=256 \mathrm{mg} / \mathrm{dl})$.

In the absence of solid consensus for the best management of coronary aneurysms, variation of strategies had been adopted amongst reported cases and decision being made based on the aneurysmal size, location, symptomatology status and severity of the coronary artery disease (4). For smaller size coronary artery aneurysms with minimal symptoms, conservative treatment including antiplatelet therapy with modification of the cardiovascular risk factors are usually sufficient (4). For those who have giant aneurysms and carrying prohibitive risk for surgery, percutaneous coronary intervention using covered stents or coil embolization had been described (4). Surgery including aneurysmal ligation or resection remains the preferred approach for giant coronary artery aneurysms because it can prevent complications and treat associated surgical conditions (5). We hence elected this method for our patient and performed coronary artery bypass grafting to the left coronary circulation with proximal and distal ligation of the giant right coronary aneurysm and bypassing the posterior descending artery-using piece of saphenous vein graft. We created a summarized flowchart demonstrating the management strategies for giant coronary artery aneurysm (Table 1)

\section{Conclusion:}

Our case highlights the importance of thorough diagnostic assessment for such coronary aneurysm before decision making plan. Combined coronary artery bypass grafting with aneurysmal exclusion is a valid option.

References:

1. Teng P, Ni C, Sun Q, Ni Y. Giant right coronary artery aneurysm mimicking a right intra-ventricular mass: a case report. J Cardiothorac Surg. 2020;15(1):17.

2. Wang H, Zhang Y, Xie Y, Wang H, Yuan J. Giant right coronary artery aneurysms presenting as a cardiac 
mass: Case report. Medicine (Baltimore). 2016;95(38):e4924.

3. Halapas A, Lausberg H, Gehrig T, Friedrich I, Hauptmann KE. Giant right coronary artery aneurysm in an adult male patient with non-ST myocardial infarction. Hellenic J Cardiol. 2013;54(1):69-76.

4. Barr J, Acharya MN, Kourliouros A, Raja SG. Technical Considerations of Giant Right Coronary Artery Aneurysm Exclusion. Case Rep Surg. 2016;2016:3795640.

5. Jha NK, Ouda HZ, Khan JA, Eising GP, Augustin N. Giant right coronary artery aneurysm- case report and literature review. J Cardiothorac Surg. 2009;4:18.

6. Nunez-Gil IJ, Alberca PM, Gonzalo N, Nombela-Franco L, Salinas P, Fernandez-Ortiz A. Giant coronary aneurysm culprit of an acute coronary syndrome. Rev Port Cardiol. 2018;37(2):203 e1- e5.

7. Saghir I, Moeen Z, Saghir G, Bangash S, Tariq S, Akter S. Giant Right Coronary Artery Aneurysm: A Case Report. Cureus. 2018;10(11):e3609.

\section{Table and Figure legends}

Table I: A flow chart summarizing the management strategies for patients with giant coronary artery aneurysm

Figure I: A. Coronary Angiogram demonstrating the giant right coronary aneurysm. B. Left coronary circulation angiography demonstrating crtitical lesions in the left coronary arteries. C. Chest CT scan demosnstrating important structures as delinated by the blue arrows. D: Intra-operative view demonstrating the giant right coronary aneurysm ligated at the proximal and distal ends

\section{Hosted file}

Table 1 Flowchart.docx available at https://authorea.com/users/318280/articles/448220-giantright-coronary-artery-aneurysm-in-the-setting-of-acute-coronary-syndrome-a-case-report

\section{Hosted file}

Figure I.pdf available at https://authorea.com/users/318280/articles/448220-giant-rightcoronary-artery-aneurysm-in-the-setting-of-acute-coronary-syndrome-a-case-report 